\title{
Infrared and Fluorescence Properties of Reduced Graphene Oxide/Regenerated Cellulose Composite Fibers
}

\author{
Xue Wang, Qinglin Yan, Xin Gao, Shuangxin Wang, Yuyu He, and Liping Zhang *
}

\begin{abstract}
To give cellulose fibers dual characteristics of warming and fluorescence, graphene oxide (GO) and fluorescent particles were simultaneously dispersed into the regenerated cellulose spinning solution through blending modification and post-reduction methods. After dry-jet wet spinning and reducing in hydrazine hydrate solution, the reduced graphene oxide (RGO)/regenerated composite fibers with different mass ratios of filler were completed. Fourier-transform infrared spectroscopy (FTIR), X-ray diffraction (XRD), scanning electron microscopy (SEM), fluorescence microscopy, and other methods were used to characterize the structure and morphology of fibers. Test results showed that the thermal stability and infrared emissivity increased gradually with the increase of GO. The crystallinity and strength of the composite fiber first increased and then decreased. This type of fiber also had luminescent properties after addition of fluorescent agent. However, when too much fluorescent agent was added, the thermal stability, infrared emissivity, crystallinity, and other properties mentioned above were affected to some extent. According to the comprehensive analysis, when the amount of GO added was $1 \mathrm{wt} \%$ and fluorescent added was $3 \mathrm{wt} \%$, respectively, the luminescence characteristic and far-infrared emissivity of the fibers were remarkably improved.
\end{abstract}

Keywords: Reduced graphene oxide; Regenerated cellulose; Infrared emissivity, Fluorescence properties

Contact information: MOE Engineering Research Center of Forestry Biomass Materials and Bioenergy, Beijing Forestry University, Beijing 100083, China; *Corresponding author: zhanglp418@ 163.com

\section{INTRODUCTION}

A major current focus on green energy revolution has forced researchers to develop renewable, degradable, and environmentally friendly fiber materials. Regenerated cellulose fiber, which is regenerated from natural cellulose, due to its environmental protection properties and easy availability, has become an ideal material that can substitute for conventional synthetic fiber (Liu et al. 2019). It has a wide range of sources, but due to the shortage of forestry resources, straw resources are now being considered as alternative cellulose sources where the cellulose content can reach up to 30 to $50 \%$. However, at present, straw has not been used effectively, except for a small part of papermaking, energy, etc. (Kim et al. 2006). Most of the straw is burned, which pollutes the environment and causes waste of resources (Liang et al. 2018). Therefore, isolating cellulose from agricultural waste to prepare regenerated cellulose fiber is one of the ways to utilize straw resources in high value. Composite fiber possesses some outstanding and comparable properties, and extends its applications for special materials (Saba et al. 2016). In recent years, fibers with functions, such as thermal insulation and fluorescence, have aroused great interest. They can be used not only in the field of textiles, but also in transportation, 
anti-counterfeiting, and in other fields. Thus, functional compounding is an important way to increase the value of cellulose fibers.

Graphene has attracted a lot of attention in recent years, especially in the area of composite materials. Since the first graphene fiber was obtained by Gao's research group of Zhejiang University in 2011, various structures and compositions of graphene fibers have been investigated, broadening the field of view for the research of excellent performing composite fibers (Zhang and Chen 2019). The unique two-dimensional sheet structure (Allen et al. 2010) and large specific area of graphene account for their remarkable mechanical and thermal properties (Mahmoudian et al. 2017). In particular, graphene is both a far-infrared absorber and a good radiator. It has a far-infrared energy storage function (Tang et al. 2014), which can produce a series of physiological responses to the human body, increasing microcirculation and metabolism. Moreover, chemically modified graphene sheets also possess these characteristics (Zhang et al. 2010), which makes it possible to produce warm fibers. To address such a property, several efforts have been applied through blending spinning and coating methods. The development of the latter method was restricted by its poor uniformity and stiff feeling (Du et al. 2016). In contrast, the former method is permanent and currently widely used in the research of synthetic fibers and viscose fibers. In this study the authors used chemical reduction and posttreatment methods to prepare reduced graphene oxide (RGO) regenerated cellulose fiber. The diluted oxygen-containing functional groups of RGO can benefit the combination with cellulose (Sawangphruk et al. 2013), it is also a simple way to realize batch production.

In addition to the far infrared properties, fluorescent fiber is another functional fiber that attracts much attention. Many attempts have been focused on the fluorescent fiber materials in recent years. Under the specific wavelength of excitation light, the fluorescent fiber can radiate fluorescence, thereby displaying the target in the dark. The fluorescent characteristic not only plays an increasingly important role in the fields of stage performance, but transportation, firefighting, and security fields (Zhang et al. 2016). To date, blending modification is mainly an approach to make fluorescent fiber, including melt spinning, solution spinning, electrospinning, etc. Among them, solution spinning possesses broad prospects (Zhao et al. 2012) because of its low temperature, convenient operation, and it does not cause the problem of thermal decomposition. In 1936, the French scientist Georges Destriau discovered that an inorganic adjunct, such as zinc sulfide, can exhibit an electroluminescent effect. Compared with organic fluorescent substances, inorganic salt fluorescent materials have the characteristics of heat resistance, good solubility, and strong stability (Wang 2015). Through mechanical blending modification and coupling with the cross-linking agent at the same time, the fluorescent substance can be added to the cellulose matrix (Liao et al. 2018).

In this study, the authors complexed different agents to a cellulose matrix, aiming to prepare the multifunctional textiles used in thermal or optical equipment. Straw pulp, which was dissolved in a novel ionic liquid to prepare a spinning solution, was selected as the raw material. After incorporating graphene oxide (GO) and $\mathrm{Zns}: \mathrm{Cu}$ fluorescent agent into the spinning solution, the chemical reduction treatment was employed after dry-jet wet spinning to achieve reduced graphene oxide/regenerated cellulose composite fibers. The effects of additives on the chemical structure, crystallinity, surface morphology, and thermal stability of fibers were discussed using Fourier transform infrared (FTIR), X-ray diffraction (XRD), scanning electron microscopy (SEM), thermogravimetric analysis (TGA), and other analyses. Moreover, the fluorescence spectroscopy, fluorescence 
microscopy, and far-infrared emissivity were also used to test the corresponding properties of the composite fiber.

\section{EXPERIMENTAL}

\section{Materials}

Straw pulp, within which the content of cellulose was more than $90 \mathrm{wt} \%$, was obtained from Jilin Chemical Fiber Group Co., Ltd. (Jilin Province, China). The novel ionic liquid was made by the authors. The graphene oxide was purchased from Leadernano Tech LLC (Shandong Province, China). The hydrazine hydrate (> 98\% (Technical grade)) was supplied by Shanghai Aladdin Bio-Chem Technology Co., Ltd. (Shanghai, China). The ethanol absolute, $\mathrm{ZnS}: \mathrm{Cu}$ fluorescent (micron size) and Titanate coupler (cp) were supplied by Beijing Chemical Works (Beijing, China).

\section{Methods}

The composite fibers were prepared used three main processes as follows:

(1) Pretreatment of fluorescent

A total of $10 \mathrm{wt} \%$ of $\mathrm{ZnS}: \mathrm{Cu}$ powder and $8.34 \mathrm{wt} \%$ of titanate coupler were added to predetermined ethanol and mechanically stirred at room temperature for $1 \mathrm{~h}$ to make them uniformly mixed. After that, evaporation was completed under vacuum at $100{ }^{\circ} \mathrm{C}$ to remove the ethanol.

(2) Preparation of RGO/regenerated cellulose fibers

The straw pulp was dissolved in ionic liquid, different amounts of graphene oxide powder was added and uniformly dispersed in the cellulose spinning solution by mechanical stirring. This solution then was transferred to glass syringes for deaeration (still) at room temperature. The method of dry-jet wet spinning was used in this study. The fiber from spinneret would first via a dry air region and then stepped into coagulation bath, thus achieved double diffusion of solvent as well as high degrees of jet-stretch ratios. After that, the GO/regenerated cellulose fibers were prepared. To obtain RGO/regenerated cellulose fiber, the above fibers were reduced by hydrazine hydrate for at least $24 \mathrm{~h}$ and finally dried at room temperature.

(3) Preparation of RGO/regenerated cellulose fluorescent fibers

Table 1. Fiber Samples with Different Additive Ratios

\begin{tabular}{|c|c|c|c|}
\hline Name & $\begin{array}{c}\text { Cellulose Concentration } \\
\text { of Spinning Solution } \\
\text { (wt\%) }\end{array}$ & $\begin{array}{l}\text { GO Addition } \\
\text { Amount (wt\%) }\end{array}$ & $\begin{array}{l}\text { Fluorescent Addition Amount } \\
\text { (wt\%) }\end{array}$ \\
\hline Z-0 & \multirow{10}{*}{ 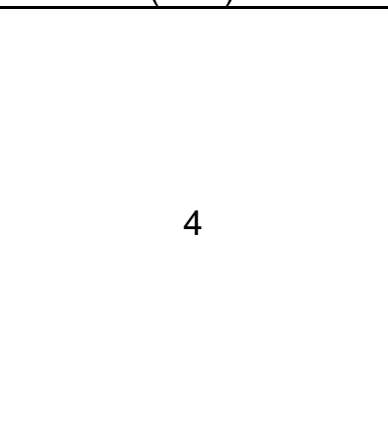 } & 0 & 0 \\
\hline Z-0.5 & & 0.5 & 0 \\
\hline Z-1 & & 1 & 0 \\
\hline Z-2 & & 2 & 0 \\
\hline Z-3 & & 3 & 0 \\
\hline $\mathrm{H}-1$ & & 1 & 1 \\
\hline $\mathrm{H}-3(\mathrm{G}-1)$ & & 1 & 3 \\
\hline $\mathrm{H}-5$ & & 1 & 5 \\
\hline G-0.5 & & 0.5 & 3 \\
\hline G-2 & & 2 & 3 \\
\hline
\end{tabular}


The pretreated fluorescent agents obtained in (1) were further added in the graphene oxide regenerated cellulose spinning solution prepared in (2), the mixture was mechanically stirred for $2 \mathrm{~h}$ to mix uniformly. The above-mentioned spinning solution was transferred to a glass syringe and the subsequent procedures were same as (2). The addition ratio of GO and fluorescent in different fibers are shown in Table 1.

\section{Characterization}

The chemical fiber structure was analyzed using a German Bruker VERTES TOV FTIR instrument (Tensor 27; Bruker, Karlsruhe, Germany). The scanning range was from 500 to $4000 \mathrm{~cm}^{-1}$. Fiber surface images were taken with SEM (Hitachi S-3400N II; Hitachi, Tokyo, Japan) after being coated with a thin layer of gold. The TGA under nitrogen was performed with a STA449F3 thermogravimetric analyzer (NETZSCH, Bavaria, Germany) to obtain a thermal decomposition curve. The temperature rise range was from room temperature to $450{ }^{\circ} \mathrm{C}$ with the rate of $20{ }^{\circ} \mathrm{C} / \mathrm{min}$. The X-ray patterns of fluorescers and fiber powder were analyzed in a reflection mode on an X-ray diffractometer (D8 ADVANCE; Bruker, Karlsruhe, Germany). The samples were tested with $\mathrm{Cu}$ Ka radiation $(k=0.154 \mathrm{~nm})$ at $40 \mathrm{kV}$ and $30 \mathrm{~mA}$ with a scan speed of $2^{\circ} / \mathrm{min}$ from $5^{\circ}$ to $50^{\circ}$. For the fluorescence properties of the fiber, the following two characterization methods were applied. Fluorescence spectroscopy was performed using a transient/steady-state fluorescence spectrometer (FLS920; Edinburgh Instruments, Bath, England). The fluorescence fiber powder was analyzed using only the stable test portion of the instrument. After the sample was stimulated at a wavelength of $365 \mathrm{~nm}$, the fluorescence emission spectrum was obtained in the range of 400 to $700 \mathrm{~nm}$. To observe the distribution of the luminescent particles on the fibers, the American fluorescent universal microscope BX61 (Olympus, Tokyo, Japan) was also used under the 100-fold bright field, which would help to tell the overall fluorescence performance of the fibers. The mechanical properties of the fibers were tested using a Zwick Roell 2.5Z stretcher (Instron, Canton, NJ, USA), whose distance between the clamps was $5 \mathrm{~cm}$ and tensile speed was fixed at $0.2 \mathrm{~mm} / \mathrm{s}$ room temperature. Each sample was tested 10 times for averaging. In the end, for the convenient detection of infrared emissivity, the spinning solution was formed into films and then solidified in a hydrazine hydrate solution for $24 \mathrm{~h}$. The film thickness was consistent. The IR-2 type dual-frequency infrared emission tester (Huaruison Co., Ltd., Beijing, China) was used with the wavelength of 8 to $14 \mu \mathrm{m}$ at $300 \mathrm{~K}$ to analyze the fiber films.

\section{RESULTS AND DISCUSSIONS}

\section{FTIR Analysis}

To verify the changes of chemical structure, FTIR spectroscopic analysis of Z-0, Z-1, and H-3 were performed, and the results are shown in Fig. 1. In the spectra of Z-0, a strong absorption band near $3400 \mathrm{~cm}^{-1}$, characteristic of free $\mathrm{O}-\mathrm{H}$ stretching vibration, was observed. The slight decrease at $3000 \mathrm{~cm}^{-1}$ was ascribed to $\mathrm{C}-\mathrm{H}$ stretching vibration. Typical peaks of the $\mathrm{C}=\mathrm{C}$ group at 1700 were exhibited. Meanwhile, water absorption also produces a certain degree of absorption ( $\mathrm{Li}$ et al. 2015). The weak band in the spectral region of 1010 to $1100 \mathrm{~cm}^{-1}$ was assigned to the stretching vibration of $\mathrm{C}-\mathrm{O}$ group (Alemdar and Sain 2008). 


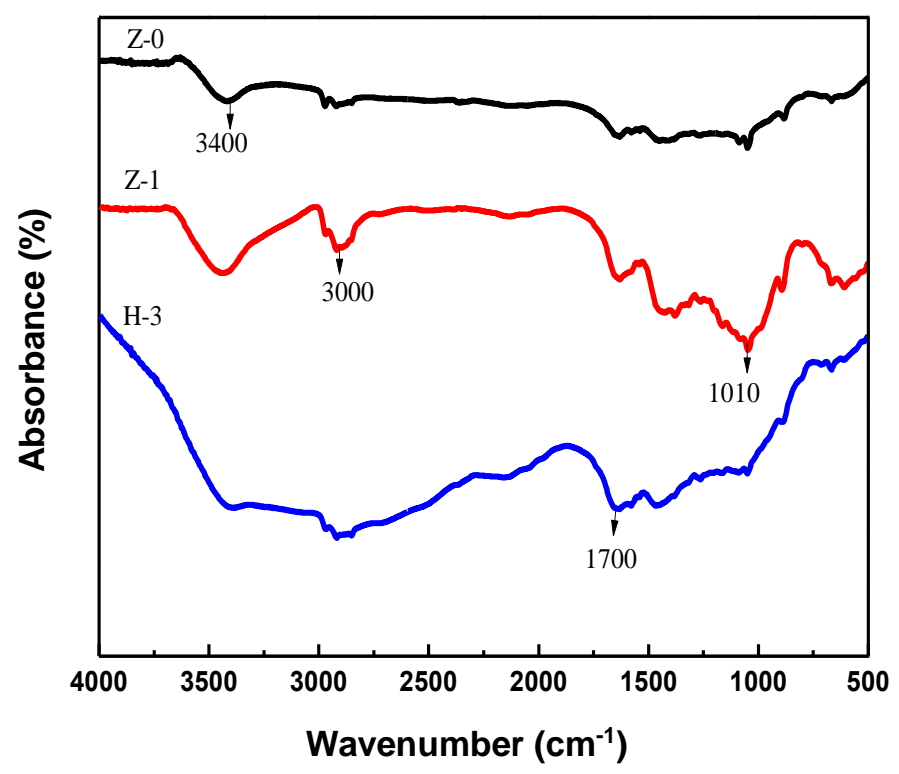

Fig. 1. FT-IR spectra of Z-0, Z-1, and H-3 fiber samples

Through comparison with Z-0, the broad and strong absorption peak around 3400 $\mathrm{cm}^{-1}$ was also exhibited in Z-1 and H-3. Moreover, the absorption peak became broad with the addition of RGO and fluorescent, which confirmed that the number of hydrogen bonds as well as intramolecular association hydrogen bonds in the system increased due to the addition agents ( $\mathrm{Lu} \mathrm{2014).} \mathrm{The} \mathrm{intensity} \mathrm{of} \mathrm{the} \mathrm{C}-\mathrm{H}$ stretching vibration peak near 3000 $\mathrm{cm}^{-1}$ was higher than that in Z-0, which may have been related to the reduction of GO. The position of the band at $1700 \mathrm{~cm}^{-1}$ was accompanied with a slight shift compared with Z-0, the intensity here increased due to $\mathrm{C}=\mathrm{C}$ bonds that not only presented in cellulose but in RGO powder. Especially in the spectra of Z-1, the absorption intensity at $1010 \mathrm{~cm}^{-1}$ was stronger than Z-0, which was attributed to the amount of oxygen-containing functional groups including C-O-C and -OH on the surface of the RGO (Li et al. 2017). In conclusion, the absorption peaks of Z-1 and H-3 fibers were similar to those of Z-0, indicating that the main groups present in the fiber samples prepared by the above method did not change.

\section{Fiber Morphology}

The SEM images of composite fibers and pure fibers are shown in Fig. 2. Cellulose fiber in Fig. 2a had an approximate cylindrical shape and smooth surface with no obvious ravines, which resulted from the absence of additives and the process of dry-jet wet spinning, thus making the formation uniform (Jiang et al. 2019). The composite fibers presented small particles and obvious ravines distributed unevenly on the surface. It was clear that the more substances added, resulted in a rougher surface. This was because GO and fluorescent particles destroyed the spinning fluency and the dense structure between macromolecules ( $\mathrm{Li}$ et al. 2017), making grains on H-3 fiber more pronounced. In addition, the solvent that was not removed may also result in the protrusion parts of the fiber surface (Bazbouz et al. 2019). The ravines on the fibers may affect the intensity of fibers and make them more easily broken. 


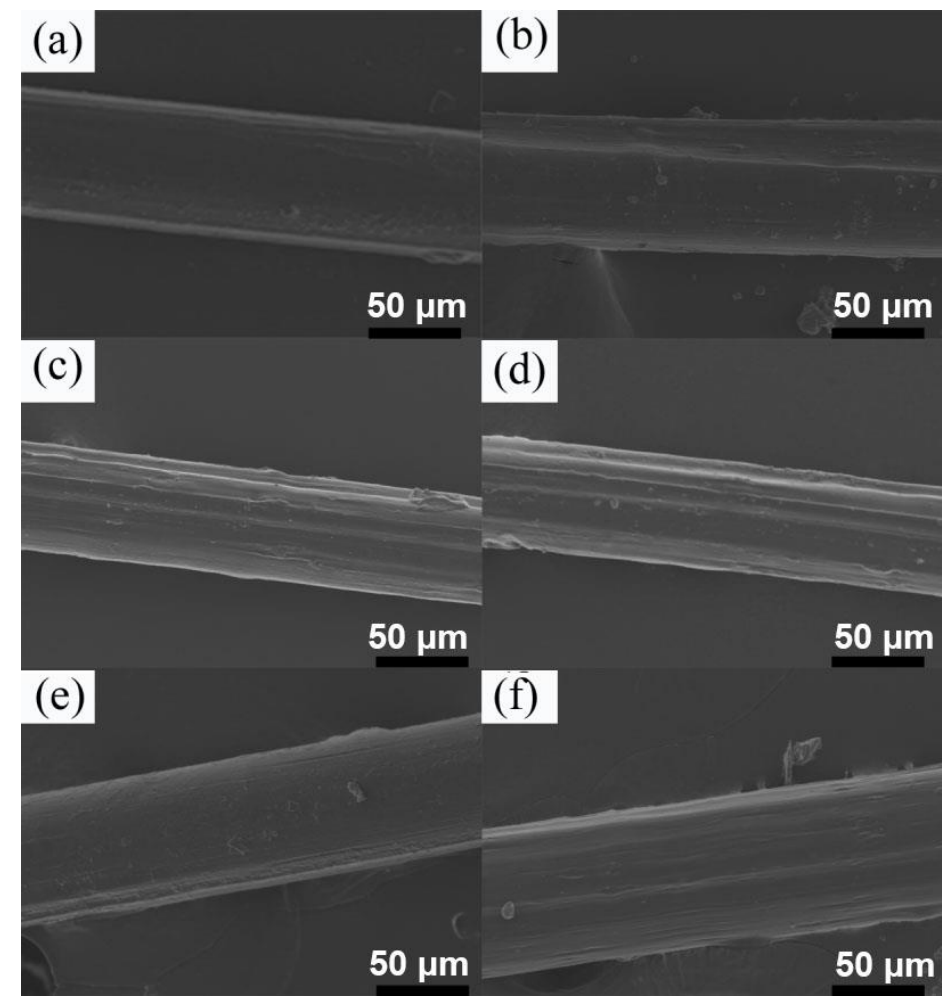

Fig. 2. SEM micrographs of fibers with different addition amounts: (a) Z-0, (b) Z-1, (c) Z-2, (d) Z3, (e) $\mathrm{H}-1$, and (f) $\mathrm{H}-3$

\section{TGA Analysis}

The TGA and derivative thermogravimetric (DTG) curves of the sample are shown in Fig. 3A and 3B. The samples display approximately $70 \%$ mass loss from 100 to $400{ }^{\circ} \mathrm{C}$. The first mass loss at approximately $100{ }^{\circ} \mathrm{C}$ was due to the evaporation of $\mathrm{H}_{2} \mathrm{O}$ and other chemicals (Yu et al. 2003). The subsequent weight loss in the range of 250 to $350{ }^{\circ} \mathrm{C}$ was the decomposition of cellulose fibers (Wang et al. 2015).

The temperature at maximum weight loss was delayed in Z-1 and H-3 compared with Z-0, the rate of loss of weight became decreased, and this trend was gradually upward with the increase of GO. Therefore, the regenerated cellulose fiber modified by RGO can significantly present better thermal stability (Greiner and Wendorff 2007). For composite fluorescent fiber (H-3), the range of degradation temperature was reduced compared to Z1 , which may have been related to the decrease in crystallinity of fiber after adding fluorescent.

\section{XRD Analysis}

The influence of RGO and fluorescent on crystallinity was examined by X-ray diffraction. The peaks of $\mathrm{ZnS}$ on JCPDS (Joint Committee on Powder Diffraction Standards) card were $28.58^{\circ}, 33.12^{\circ}$, and $47.61^{\circ}$, corresponding to (111), (200), and (220) planes, respectively (Espinosa et al. 2019). Figure 4 shows the X-ray diffraction patterns of the fluorescent and fibers in this study. Compared to the study researched by Espinosa's group, the visible peaks were approximately at the same location, thus clarifying that this type of fluorescent is $\mathrm{ZnS}$. The characteristic high peak and the sharp shape in the XRD patterns illustrate that the fluorescent atoms are arranged more regularly and a fine crystal structure is formed (Wang 2014). 


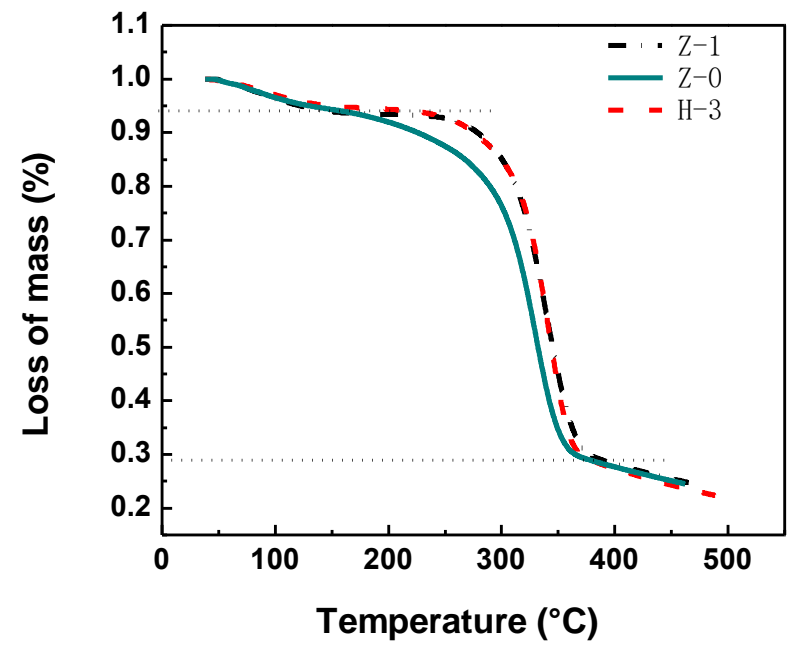

(A)

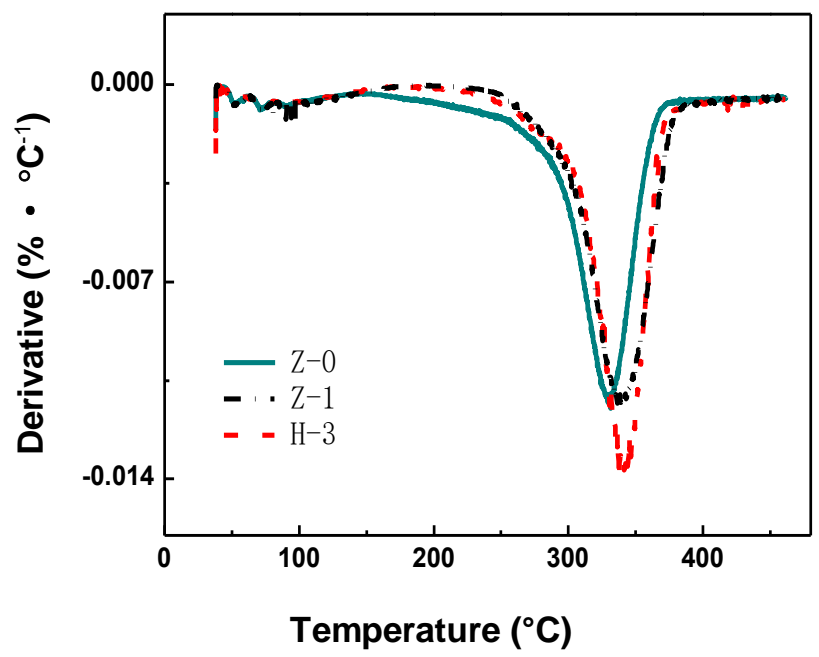

(B)

Fig. 3. A) TGA of Z-0, Z-1, and H-3 fiber sample; B) DTG of Z-0, Z-1, and $H-3$ fiber sample

From the XRD patterns of Z-0, Z-1, Z-3, and H-3, the authors found that the diffraction peaks of different fibers located at $12.19^{\circ}, 20.18^{\circ}$, and $20.76^{\circ}$ can be assigned to cellulose II (Zhang et al. 2018). This indicated the cellulose structure was converted from cellulose I to cellulose II after being regenerated, and the peaks became broad and weak due to the decrease of crystallinity. For the XRD of RGO reduced by hydrazine hydrate, a broad reflection peak centered at $24^{\circ}$ is observed in the pattern researched by Zhang's group (Zhang et al. 2010). However, its characteristic peaks are not clearly shown in this pattern. This may have a certain degree of coincidence with the diffraction peak near $22.5^{\circ}$ of cellulose II. In addition, the content of RGO in the solution is low and the diffraction intensity might be too weak to present (Li et al. 2019a). The crystallinity of Z$0, \mathrm{Z}-1$, and Z-3 were $35.9 \%, 40.2 \%$, and $31.2 \%$, respectively, confirming that the crystallinity can be improved with the addition of a certain amount of GO (Ouyang et al. 2013). However, the crystallinity decreased with the increase of the GO and fluorescent when the content of additives exceeded $1 \mathrm{wt} \%$. This may have been because the particles gathered may have destroyed the hydrogen bond between the cellulose macromolecules, and thus the dense structure of the cellulose would be damaged. 


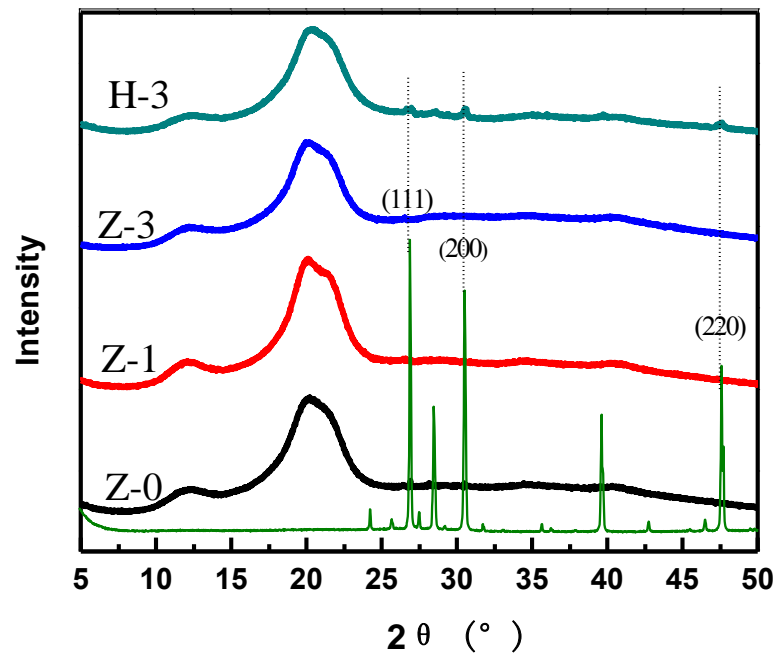

Fig. 4. X-ray diffraction pattern of fluoresce complex and Z-0, Z-1, Z-3, and H-3 fiber sample

H-3 exhibited new diffraction peaks at $26.99^{\circ}$ and $34.84^{\circ}$, demonstrating that the fluorescent was successfully added to the composite fiber. Furthermore, Compared with Z1 fiber, the crystallinity of H-3 sample decreased to $26.1 \%$, which may have been influenced by the fluorescent and GO added during crystal growth, resulting in a decrease in crystallinity (Zhang et al. 2003). Therefore, when fluorescent is added to the fiber, it may prevent the action of RGO from increasing the crystallinity to some extent.

\section{Fluorescence Spectra Analysis}

Figure 5 is the fluorescence spectrum of $\mathrm{H}-1, \mathrm{H}-3$, and $\mathrm{H}-5$ fibers. It has been studied that after excitation at a wavelength of $365 \mathrm{~nm}, \mathrm{~S}^{2-}$ in $\mathrm{ZnS}: \mathrm{Cu}$ fluorescent can have an emission maxima at approximately $430 \mathrm{~nm}$ and a broad blue-green fluorescence emission peak at approximately $530 \mathrm{~nm}$ (Small and Johnston 2008).

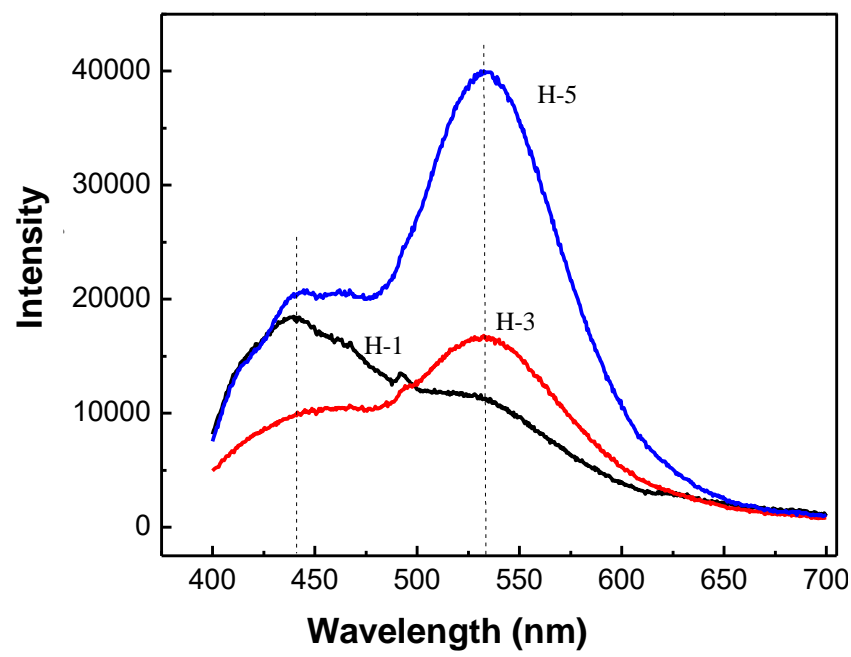

Fig. 5. Fluorescence spectra of fluorescence cellulose fiber with different concentrations

In the authors' research, all three curves had strong emissions at approximately 430 $\mathrm{nm}$ at different levels. H-3 and H-5 fibers also showed the highest intensity at approximately $530 \mathrm{~nm}$, illustrating that the result was the same as the spectra measured in 
the current study, verifying the fluorescent agent prepared in this study was $\mathrm{ZnS}: \mathrm{Cu}$ fluorescent (Cui et al. 2018). It is worth noting that, in the location of $530 \mathrm{~nm}$, the emission intensity tended to increase distinctly upon the increase of fluorescent, which suggests that the intensity at this wavelength was positively correlated with the concentration of fluorescent. However, the intensity had no obvious relationship at $430 \mathrm{~nm}$ with the amount of fluorescent, which may have been related to the dispersion degree of fluorescent and RGO. In general, the composite fibers had different degrees of fluorescence emission capability after addition of $\mathrm{ZnS}: \mathrm{Cu}$ fluorescent.

\section{Fluorescence Microscopy Characterization}

Figure 6 shows the fluorescent microscope images before (a) and after (b) the treatment by coupling agent. The fluorescent powder showed obviously under blue light optical filter. Before treated with coupling agent, the fluorescent agent was finely granulated and densely dispersed in the field of view. Due to the inorganic characteristic, it was difficult for the fluorescent to uniformly disperse into the organic cellulose spinning solution, thus affecting the fluorescence properties of the fiber. Herein, after addition of coupling agent, most of the dispersed small particles became gathered. This was because low molecular mass chains that existed in the coupling agent reacted with the inorganic agents to form a molecular layer, thereby linking to the inorganic molecule. Other parts of the long chain intertwine with the cellulose polymer (Guo et al. 2003). In this way, the inorganic substance and the cellulose are tightly integrated by the 'media' coupling agent, so that the fluorescent can be uniformly dispersed in the cellulose spinning solution.
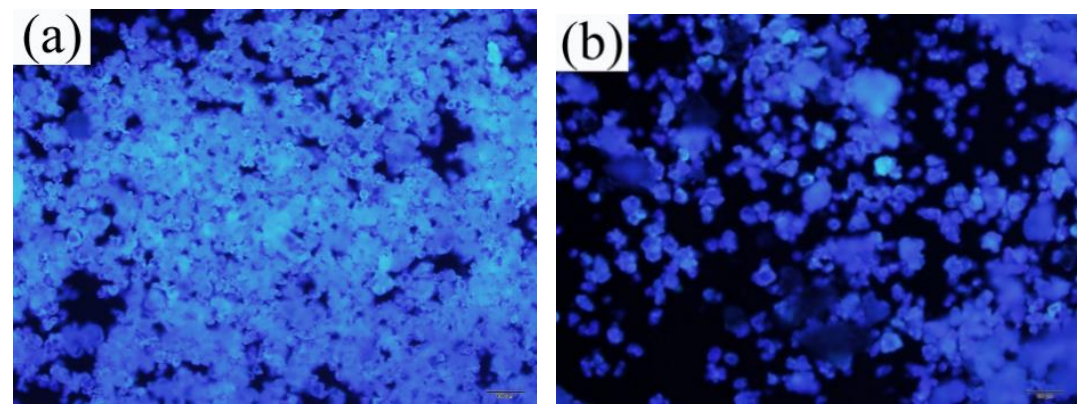

Fig. 6. Fluorescence microscopy characterization (a) before and (b) after pretreatment

Figure 7 shows the fluorescence microscope images of $\mathrm{H}-1, \mathrm{H}-3$, and $\mathrm{H}-5$ cellulose fibers containing different fluorescent. It is well known that the larger aggregates are the main factors that influence the mechanical properties of fiber (Zhang et al. 2016). To pursue the dual characteristics of mechanical and fluorescent properties, one of the key methods is to prevent the agglomeration of fluorescent powder and improve the compatibility between fluorescent powder and the cellulose matrix. It can be seen from Fig. 7(a) that there were fewer fluorescent particles in the field of view, and the fluorescence characteristic was not obvious when the fluorescent was under $1 \mathrm{wt} \%$. By contrast, in $\mathrm{H}-3$ the $3 \mathrm{wt} \%$ addition of couple agent was dispersed homogeneously, as shown in Fig. 7(b), and the fluorescence characteristic was clearly observed. When fluorescent exceeded $5 \mathrm{wt} \%$, the fiber was partially bright and the brightness was uneven; moreover, the self-agglomeration of the fluorescent is observed (Zhao et al. 2012). In summary, the composite fiber with $3 \mathrm{wt} \%$ fluorescent possessed better fluorescence characteristic. 

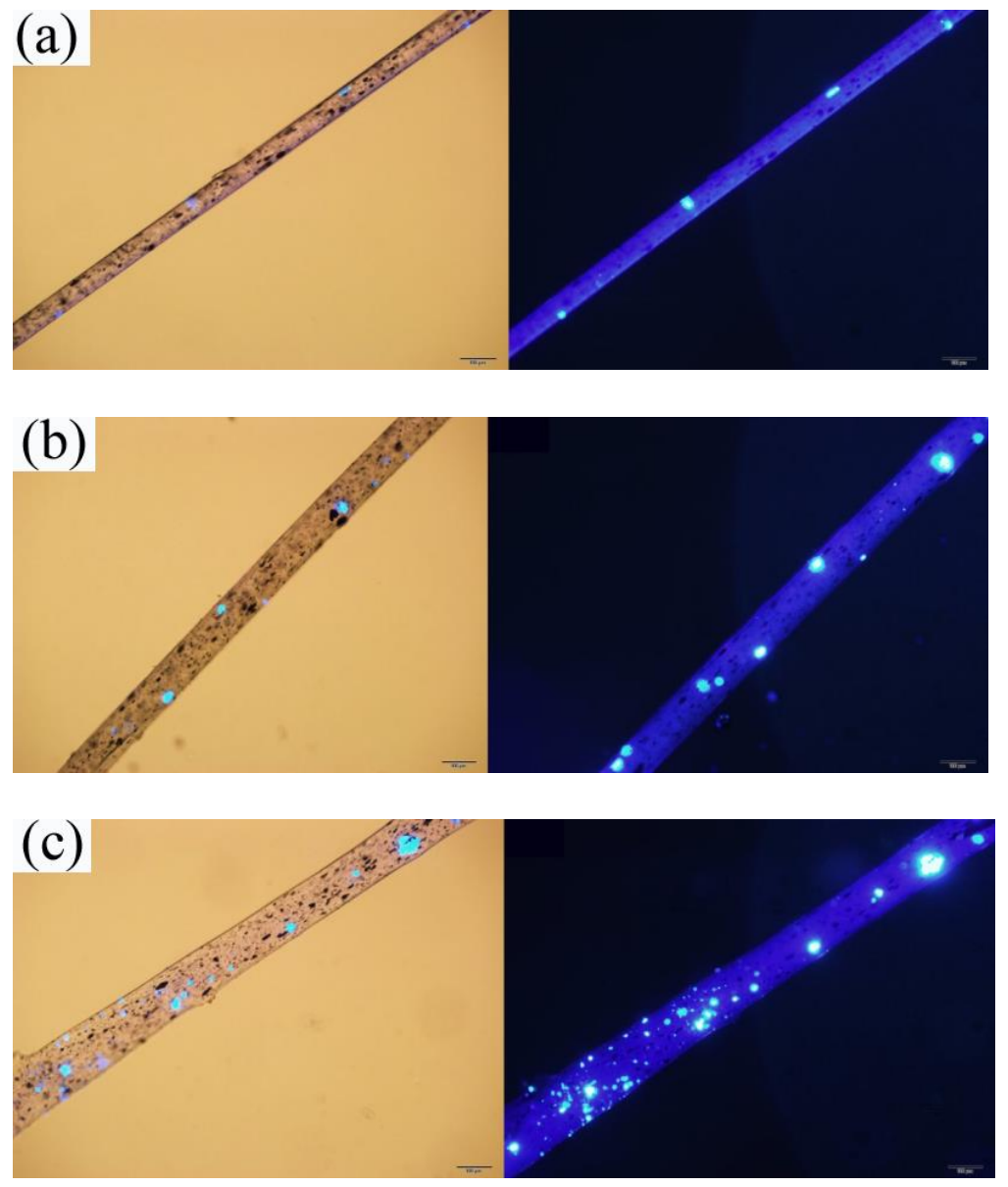

Fig. 7. Fluorescence microscopy characterization of composite fibers: (a) $\mathrm{H}-1$, (b) $\mathrm{H}-3$, and (c) $\mathrm{H}-$ 5

\section{Mechanical Properties}

The mechanical properties of fibers at room temperature including tensile strength and elongation at break are listed in Table 2. As the proportion of RGO increased, the intensity of the composite fibers first increased and then decreased.

Table 2. Physical Material Property of Fibers with Different Addition Amount

\begin{tabular}{|c|c|c|}
\hline Name & $\begin{array}{c}\text { Intensity(cN/dtex) } \\
(1 \mathrm{dtex}=1 \mathrm{~g} / 10000 \mathrm{~m})\end{array}$ & Elongation at Break (\%) \\
\hline Z-0 & 2.230 & 14.4 \\
\hline Z-1 & 2.294 & 10.8 \\
\hline Z-2 & 2.282 & 10.0 \\
\hline G-0.5 & 1.941 & 5.08 \\
\hline H-3 & 2.030 & 6.40 \\
\hline
\end{tabular}

When fibers were blended with $1 \mathrm{wt} \% \mathrm{GO}$, the material could withstand a stress as high as $2.294 \mathrm{cN} / \mathrm{dtex}$, which was $3 \%$ higher than that of the pure regenerated cellulose fiber, indicating appropriate RGO will improve the strength of the fiber (Li et al. 2019b). The high fracture strain is attributed to the RGO with better performance as well as the good coherence between the regenerated cellulose matrix and RGO (Li et al. 2017). However, when content of GO exceeded $1 \mathrm{wt} \%$, the degree of crystallinity was lowered 
due to agglomeration between GO particles, resulting the decrease in intensity. Fluorescent particles have the same effect on fibers, so the dense crystal structure of the regenerated cellulose is further destroyed due to the self-aggregation of the fluorescer particles; thus the intensity of $\mathrm{G}$ and $\mathrm{H}$ groups was lower than that of the $\mathrm{Z}$ group. Compared to Z-1, $\mathrm{H}-3$ had a reduction of approximately $13 \%$. In addition, the tendency of elongation at break continued to decrease. Therefore, the addition of additives may cause local brittleness and reduce the toughness of fiber (Luzi et al. 2016).

\section{Infrared Emissivity Analysis}

Figure 8 shows the relationship between additives content and infrared emissivity of composite fibers. It shows noticeable enhancement of the emissivity value with respect to the bare cellulose fiber. Graphene, which has strong light absorption ability, can absorb light in all wavelength bands and output in the form of far infrared energy (Zhao et al. 2019). Many hydroxyl groups and $\mathrm{C}-\mathrm{H}$ bonds on cellulose surface can form $\mathrm{CH}-\pi$ interaction with graphene, thus improving the performance of cellulose. So the far-infrared emissivity of the composite fiber membrane was obviously higher than that of the regenerated cellulose fiber membrane. When the content of GO increased, the value of farinfrared emissivity increased as well. When GO was $1 \mathrm{wt} \%$, the far-infrared emissivity reached a maximum of 0.96 . While the graphene content exceeded $1 \mathrm{wt} \%$, the tendency was constant and even lower.

Concerned with the conclusion obtained in the fluorescence characterization above, to analyse the influence of fluorescent, $3 \mathrm{wt} \% \mathrm{ZnS}: \mathrm{Cu}$ fluorescent was further added to the above-mentioned fiber spinning solution to investigate the far-infrared emission properties of the fiber membrane, as also shown in Fig. 8.

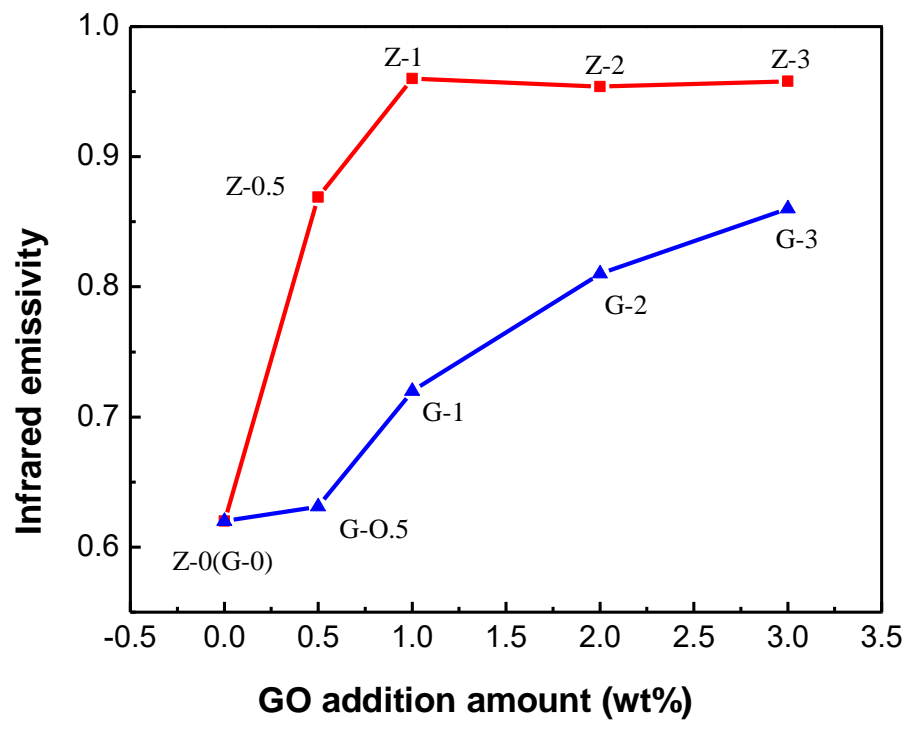

Fig. 8. The infrared emissivity of cellulose membrane

The emissivity showed an obvious reduction tendency compared with the line on the top, which indicates that the improvement on far-infrared emissivity by the function of RGO will be inhibited by fluorescent agent to some extent. However, compared to pure regenerated cellulose at 0.62 , the emissivity will gradually increase to 0.72 when the content of GO reaches $1 \mathrm{wt} \%$. The presence of GO took an essential effect on infrared 
emissivity even if other agents were added to the matrix. The addition of the fluorescent will delay the maximum peak. According to mechanical analysis, if GO content continuously increased, the crystallinity of the fiber will be lowered to affect the properties such as intensity and elongation at break. In addition, the far-infrared emissivity of the film is also related to the surface condition such as roughness, color, and thickness of the film.

\section{CONCLUSIONS}

The regenerated cellulose fibers were modified by the combination of graphene oxide (GO) and fluorescent agent through post-reduction treatment, the effects of different contents of additives on the infrared emissivity and fluorescence characteristics were investigated.

1. To prepare reduced graphene oxide (RGO)/regenerated cellulose, GO was only added to the spinning solution. With the increase of GO addition, the mechanical strength, crystallinity, thermal stability, and infrared emissivity of the fiber were improved. When the addition amount was $1 \mathrm{wt} \%$, the far-infrared emissivity of composite fiber reached the peak at 0.96 . When the GO content was more than $1 \mathrm{wt} \%$, the above properties of the fiber tended to decrease due to the agglomeration of addition particles.

2. If GO and fluorescent agent were added to spinning solution simultaneously, fibers containing $3 \mathrm{wt} \%$ of fluorescent agent had good luminescent properties, but the $\mathrm{ZnS}: \mathrm{Cu}$ fluorescent agent hindered the improvement of thermal stability, crystallinity, mechanical properties, infrared emissivity, etc., so that their performance values were slightly lower than single functional graphene fiber. In all, in the experimental range, when the fluorescent and GO content was $3 \mathrm{wt} \%$ and $1 \mathrm{wt} \%$, respectively, the fiber emitted light uniformly, and the far-infrared emissivity increased 16\% compared with the pure regenerated cellulose fiber. Other properties, such as crystallinity, were also greatly improved.

3. Through modification of the fibers by blending two additives into the cellulose matrix, the fibers can simultaneously obtain the characteristics of fluorescence and infrared emissivity, which makes it a possible way to prepare multi-functional fibers used in special clothing and sensors. On the basis of studying the influence of addition content, the additive size, choice of the dispersion system, and the modification method can also effect the performance of fibers.

\section{REFERENCES CITED}

Alemdar, A., and Sain, M. (2008). "Isolation and characterization of nanofibers from agricultural residues - Wheat straw and soy hulls," Bioresource Technology 99(6), 1664-1671. DOI: 10.1016/j.biortech.2007.04.029

Allen, M. J., Tung, V. C., and Kaner, R. B. (2010). "Honeycomb carbon: A review of graphene," Chemical Reviews 110(1), 132-145. DOI: 10.1021/cr900070d

Bazbouz, M. B., Taylor, M., Baker, D., Ries, M. E., and Goswami, P. (2019). "Dry-jet wet electrospinning of native cellulose microfibers with macroporous structures from 
ionic liquids," Journal of Applied Polymer Science 136(10), Article number 47153. DOI: 10.1002/app.47153

Cui, T. T., Zhu, Z. J., Cheng, R., Tong, Y. L., Peng, G., Wang, C. F., and Chen, S. (2018). "Facile access to wearable device via microfluidic spinning of robust and aligned fluorescent microfibers," ACS Applied Materials \& Interfaces 10(36), 30785-30793. DOI: $10.1021 /$ acsami.8b11926

Du, M. Z., Tian, M. W., and Qu, L. J. (2016). "Research progress of far-infrared textiles and novel graphene far-infrared functional textiles," Journal of Chengdu Textile College 33(4), 132-137. DOI: 10.3969/j.issn.1008-5580.2016.04.028

Espinosa, E., Rol, F., Bras, J., and Rodriguez, A. (2019). "Production of lignocellulose nanofibers from wheat straw by different fibrillation methods. Comparison of its viability in cardboard recycling process," Journal of Cleaner Production 239, WOS:000487237100103. DOI: 10.1016/j.jclepro.2019.118083

Greiner, A., and Wendorff, J. H. (2007). "Electrospinning: A fascinating method for the preparation of ultrathin fibres," Angewandte Chemie-International Edition 46(30), 5670-5703. DOI: 10.1002/anie.200604646

Guo, Y. L., Zhang, S. R., and Li, L. P. (2003). "Types, characteristics and applications of coupling agents," China Rubber Industry 11, 692-696.

Jiang, Z. M., Tang, L., Gao, X., Zhang, W. T., Ma, J. W., and Zhang, L. P. (2019). "Solvent regulation approach for preparing cellulose-nanocrystal-reinforced regenerated cellulose fibers and their properties," ACS Omega 4(1), 2001-2008. DOI: 10.1021/acsomega.8b03601

Kim, M. S., Baek, J. S., Yun, Y. S., Sim, S. J., Park, S., and Kim, S. C. (2006). "Hydrogen production from Chlamydomonas reinhardtii biomass using a two-step conversion process: Anaerobic conversion and photosynthetic fermentation," International Journal of Hydrogen Energy 31(6), 812-816. DOI: 10.1016/j.ijhydene.2005.06.009

Li, C. L., Ma, J. Z., Qin, C. M., Qu, L. J., and Tian, M. W. (2015). "Preparation and properties study of graphene/regenerated cellulose composite fiber," Knitting Industry 6, 6-8.

Li, X. M., Zhang, K. K., Shi, R., Ma, X. M., Tan, L. W., Ji, Q., and Xia, Y. Z. (2017). "Enhanced flame-retardant properties of cellulose fibers by incorporation of acidresistant magnesium-oxide microcapsules," Carbohydrate Polymers 176, 246-256. DOI: 10.1016/j.carbpol.2017.08.096

Li, N., Ma, Z. K., Chen, M., Song, H. H., Li, A., and Jia, Y. R. (2017). "Structures and performance of graphene/polyimide composite graphite fibers," Journal of Materials Engineering 45(9), 31-37. DOI: 10.11868/j.issn.1001-4381.2016.000251

Li, S., Chen, Z. F., Rao, Z. Y., Wang, F., Wu, C., and Ye, X. L. (2019a). "The preparation and research of reduced graphene oxide/glass composite fiber," Journal of Engineered Fibers and Fabrics 14, WOS:000495663600001. DOI: $10.1177 / 1558925019883105$

Li, X., Li, H. C., You, T. T., Wu, Y. Y., Ramaswamy, S., and Xu, F. (2019b). "Fabrication of regenerated cellulose membranes with high tensile strength and antibacterial property via surface amination," Industrial Crops and Products 140, Article ID 111603. DOI: $10.1016 /$ j.indcrop.2019.111603

Liang, L. F., Xin, M. Y., and Wang, H. (2018). "Research of extraction of rice straw cellulose and feasibility of fiber formation," Journal of Textile Science and Engineering 35(3), 86-89. DOI: 10.3969/j.issn.2096-5184.2018.03.019 
Liao, M., Sun, H., Zhang, J., Wu, J. X., Xie, S. L., Fu, X. M., Sun, X. M., Wang, B. J., and Peng, H. S. (2018). "Multicolor, fluorescent supercapacitor fiber," Small 14(43), Article ID 1702052. DOI: 10.1002/smll.201702052

Liu, W., Liu, S., Liu, T., Liu, T., Zhang, J., and Liu, H. (2019). "Eco-friendly postconsumer cotton waste recycling for regenerated cellulose fibers," Carbohydrate Polymers 206, 141-148. DOI: 10.1016/j.carbpol.2018.10.046

Lu, J. T. (2014). The Preparation and Performance Study of Skin Repair Materials Based on Regenerated Cellulose, Master's Thesis, Jiangnan University, Jiangsu, China.

Luzi, F., Fortunati, E., Jiménez, A., Puglia, D., Pezzolla, D., Gigliotti, G., Kenny, J. M., Chiralt, A., and Torre, L. (2016). "Production and characterization of PLA_PBS biodegradable blends reinforced with cellulose nanocrystals extracted from hemp fibres," Industrial Crops and Products 93, 276-289. DOI: 10.1016/j.indcrop.2016.01.045

Mahmoudian, S., Sazegar, M. R., Afshari, N., and Wahit, M. U. (2017). "Graphene reinforced regenerated cellulose nanocomposite fibers prepared by Lyocell process," Polymer Composites 38(S1), E81-E88. DOI: 10.1002/pc.23864

Ouyang, W. Z., Sun, J. H., Memon, J., Wang, C., Geng, J. X., and Huang, Y. (2013). "Scalable preparation of three-dimensional porous structures of reduced graphene oxide/cellulose composites and their application in supercapacitors," Carbon 62, 501509. DOI: 10.1016/j.carbon.2013.06.049

Saba, N., Jawaid, M., Alothman, O. Y., and Paridah, M. T. (2016). "A review on dynamic mechanical properties of natural fibre reinforced polymer composites," Construction and Building Materials 106, 149-159. DOI: 10.1016/j.conbuildmat.2015.12.075

Sawangphruk, M., Srimuk, P., Chiochan, P., Krittayavathananon, A., Luanwuthi, S., and Limtrakul, J. (2013). "High-performance supercapacitor of manganese oxide/reduced graphene oxide nanocomposite coated on flexible carbon fiber paper," Carbon 60, 109-116. DOI: 10.1016/j.carbon.2013.03.062

Small, A. C., and Johnston, J. H. (2008). "Novel hybrid materials of cellulose fibres and doped ZnS nanocrystals," Current Applied Physics 8(3-4), 512-515. DOI: 10.1016/j.cap.2007.10.046

Tang, L. B., Ji, R. B., Li, X. M., Bai, G. X., Liu, C. P., Hao, J. H., Lin, J. Y., Jiang, H. X., Teng, K. S., Yang, Z. B., et al. (2014). "Deep ultraviolet to near-infrared emission and photoresponse in layered n-doped graphene quantum dots," ACS Nano 8(6), 63126320. DOI: 10.1021/nn501796r

Wang, J. J. (2014). Preparation, Characterization and Nonlinear Optical Properties of Graphene-Carboxymethyl Cellulose Nano-composite Materials, Master's Thesis, Fuzhou University, Fujian, China.

Wang, P., Tawiah, B., Tian, A., Wang, C., Zhang, L., and Fu, S. (2015). "Properties of alginate fiber spun-dyed with fluorescent pigment dispersion," Carbohydrate Polymers 118, 143-149. DOI: 10.1016/j.carbpol.2014.11.028

Wang, R. (2015). Study on the Preparation and Properties of the Fluorescent Fibers, Master's Thesis, Donghua University, Shanghai, China.

Yu, J. G., Yu, H. G., Cheng, B., Zhao, X. J., Yu, J. C., and Ho, W. K. (2003). "The effect of calcination temperature on the surface microstructure and photocatalytic activity of TiO2 thin films prepared by liquid phase deposition," Journal of Physical Chemistry $B$ 107(50), 13871-13879. DOI: 10.1021/jp036158y 
Zhang, H. H., Wang, R., Yang, G. S., Xu, Y. Y., and Shao, H. L. (2016). "UV-excitable fluorescent poly(lactic acid) fibers," Polymer Engineering and Science 56(4), 373 379. DOI: $10.1002 /$ pen. 24262

Zhang, H. R., Huang, S. P., Gong, J. H., Wu, J., and Xuan, Lu. (2003). "Property and structure of fluorescence fiber," Shanghai Textile Science and Technology 2, 13-15. DOI: 10.16549/j.cnki.issn.1001-2044.2003.02.004

Zhang, K., Zhang, L. L., Zhao, X. S., and Wu, J. S. (2010). "Graphene/polyaniline nanofiber composites as supercapacitor electrodes," Chemistry of Materials 22(4), 1392-1401. DOI: $10.1021 / \mathrm{cm} 902876 \mathrm{u}$

Zhang, Y. C., Jiang, Y., Han, L., Wang, B., Xu, H., Zhong, Y., Zhang, L., Mao, Z., and Sui, X. (2018). "Biodegradable regenerated cellulose-dispersed composites with improved properties via a pickering emulsion process," Carbohydrate Polymers 179, 86-92. DOI: 10.1016/j.carbpol.2017.09.065

Zhang, Y. Y., and Cheng, Q. F. (2019). "Progress in research of graphene nanocomposite fibers," Materials China 38(1), 49-57. DOI: 10.7502/j.issn.1674-3962.2019.01.06

Zhao, C. X., Gao, X. N., and Feng, L. Q. (2012). "Application of luminescent fiber on knitted fabric," Jiangsu Silk 5, 35-38.

Zhao, L. Y., Zhang, R. Y., Deng, C. Y., Peng, Y. X., and Jiang, T. (2019). “Tunable infrared emissivity in multilayer graphene by ionic liquid intercalation," Nanomaterials 9(8), Article number 1096. DOI: 10.3390/nano9081096

Article submitted: January 17, 2020; Peer review completed: April 3, 2020; Revised version received and accepted: April 20, 2020; Published: April 27, 2020.

DOI: $10.15376 /$ biores.15.2.4434-4448 